\title{
Polarization oscillations in a media with inhomogeneous anisotropy and gyrotropy
}

\author{
Ara L. Aslanyan, Levon S. Aslanyan”, Stella K. Nazaryan. \\ Department of Physics, Yerevan State University, 1 Alex Manoogian, Yerevan 025, Armenia
}

ABSTRACT

The problem of polarized light propagation in the media with weak inhomogeneous anisotropy and gyrotropy (natural and induced) is considered. It is shown that the polarization state in such media undergoes spatial oscillations, which amplitude is maximum when changes of dielectric permeability and gyrotropy are same.

Keywords: polarization, inhomogeneous, anisotropy, gyrotropy, optical-mechanical analogy.

\section{INTRODUCTION}

The light wave polarization state is changes, when it propagates in an inhomogeneous media. Therefore, there is a possibility of managing the light polarization state by induced inhomogeneity (with the external influence). That is why the study of such spatial dynamics of the lights polarization state in different media has a great practical importance. Consideration of light polarization state spatial dynamics in inhomogeneous anisotropic media is of a great interest in various areas of physics. Propagation of light waves in photonic crystals and liquid crystals, light propagation through the cholesteric liquid crystal layer containing defects managed by external electric field, splitting of particle beams with spin in magnetic fields, polarization technique application in a biomedical research - this is the incomplete list of problems concerning the theory of wave processes in weak anisotropic media [1-4]. On the other hand, one can control the polarization state of light by changing parameters of a media with the help of external influence. That's why the study of peculiarities of the propagation of a plane monochromatic wave in media with spatial inhomogeneity (in particular, of anisotropy and gyrotropy) is of great interest also in the various fields of physics. The analytical solution of the wave equation in such media is connected with certain difficulties even for slow change of media parameters and application of the approached methods. And here analogy between mechanics and optics, which has played a significant role in the development of modern concepts of physics, comes to the aid [5]. The resonance phenomena in two level systems can serve as examples [6]. These include the tasks associated with the propagation of the polarized waves in media with spatial inhomogeneous anisotropy and gyrotropy. They are described mathematically by the same equations that describe the behavior of a two level system in stationary fields $[7,8]$. The above mentioned analogy also allows using the welldeveloped techniques from the theory of quasi-resonant interaction of radiation with a two-level atom. This is confirmed by recently published works. In particular, in $[9,10]$ adiabatic rotation and achromatic polarization conversion in inhomogeneous anisotropic media are studied. The treatment is based on the analogy of the equations describing the spatial

*leon@ysu.am; phone; +37493556383

Photonics and Micro- and Nano-structured Materials 2011, edited by Rafael Kh. Drampyan,

Proc. of SPIE Vol. 8414, 841411 - (C) 2012 SPIE · CCC code: 0277-786X/12/\$18 - doi: 10.1117/12.923189

Proc. of SPIE Vol. $8414841411-1$ 
dynamics of polarization in an inhomogeneous anisotropic media and the Schrödinger equation, which describes the coherent laser excitation of a three-level atom (so-called $\Lambda$ systems). In the special case of the adiabatic polarization sequence for smooth changes in the media the possibility of almost independent from wavelength conversion of circularly polarized light into linearly polarized light are discussed in [11] by numerical analysis of the wave equation. A similar problem was solved in [12], but in the approximation of geometrical optics. Results are fully consistent with numerical simulations [11]. In [13] the gyroscopic model for analyzing the evolution of the polarization state of light in weakly inhomogeneous anisotropic media with homogeneous gyrotropy is used. In these studies propagation of polarized light either in inhomogeneous anisotropic media, or in a pure gyrotropic media as well as in the case of presence of these two factors (including the presence of spatial inhomogeneity of one of these factors) are treated with unified position.

The purpose of the present work is to analyze the light polarization spatial dynamics in weakly inhomogeneous anisotropic media with inhomogeneous gyrotropy within the analogy between mechanics and optics, which allows to reduce the system of the equations, describing Stokes vector behavior, to the Bloch`s equations.

\section{BASIC MODEL}

For normal incidence on the media with inhomogeneity of anisotropy and gyrotropy is not difficult to obtain a system of coupled equations for two-dimensional Jones vector $\vec{E}=\left(E_{x}, E_{y}\right)^{T}$, describing the polarization state of light.

Consider the plane monochromatic wave normally incident on an optically uniaxial media, the major axes of which are oriented along the axes of the chosen laboratory coordinate system. We also assume that the media has optical activity. The inhomogeneity of anisotropy may be caused by the presence of external influence, and inhomogeneity of optical activity - by the spatial inhomogeneity of the chiral molecules concentration. The dielectric permeability of this media

$$
\hat{\varepsilon}(z)=\hat{\varepsilon}_{0}(z)+i \hat{g}(z) .
$$

Here $\hat{\varepsilon}_{0}(z)$ is tensor characterizing the inhomogeneous birefringence, and $\hat{g}(z)$-gyrotropy. Note, that we neglect absorption and the magnetic permeability is taken to be unity. Then for the Jones vector $\vec{E}=\left(E_{x}, E_{y}\right)^{T}$, describing the light polarization state can be easily obtained the following equation [13]:

$$
\frac{d^{2}}{d \xi^{2}} \vec{E}(\xi)+\hat{\varepsilon}(\xi) \vec{E}(\xi)=0,
$$

where $\hat{\varepsilon}$ is two-dimensional permittivity tensor of the media, and $\xi=(\omega / c) z$-dimensionless coordinate along the propagation of light. If the spatial inhomogeneity of the media is weak, the amplitude of the wave is a slowly varying function from coordinate $\xi$. With that the solution of (2) can be written in following form $[7,13]$

$$
\vec{E}(\xi)=\vec{E}_{0}(\xi) \exp \{i \Phi(\xi)\}
$$

In (3) $\vec{E}_{0}(\xi)$ is slowly varying complex amplitude and the phase factor is $\Phi(\xi)=\int n(\xi) d \xi$, where $n(\xi)=$ $\sqrt{\left(\varepsilon_{x x}+\varepsilon_{y y}\right) / 2}$. Substituting the solution of the desired form (3) in equation (2) and considering the slowness of the 
change of $\vec{E}_{0}(\xi)$ (neglecting the small quantity $d^{2} \vec{E}_{0} / d \xi^{2}$ ), after simple transformations the following vector equation is obtained:

$$
i \frac{d}{d \xi} \vec{J}(\xi)=\widehat{H}(\xi) \vec{J}(\xi)
$$

Here $\vec{J}(\xi)=\sqrt{\mathrm{n}(\xi)} \vec{E}_{0}(\xi)$, and $\widehat{H}(\xi)=\left\{n^{2}(\xi) \hat{I}-\hat{\varepsilon}(\xi)\right\} / 2 n(\xi), \hat{I}$ - is the unit matrix. We expand the matrix into the Pauli matrices $\widehat{\sigma}_{l}[3]$ :

$$
\widehat{H}=\frac{1}{2} \sum_{i=o}^{3} \omega_{i} \widehat{\sigma}_{l}
$$

where

$$
\omega_{i}=\operatorname{Sp}\left(\widehat{H} \widehat{\sigma}_{l}\right) .
$$

Transition to a Stokes vector is carried out as follows $[4,7]$

$$
\hat{S}=\vec{E}^{+} \hat{\sigma} \vec{E}=\frac{1}{n(\xi)} \vec{J}^{+} \hat{\sigma} \vec{J}
$$

Substituting the formula (6) for the matrix $\widehat{H}$, using (5) and (8), for propagation in a nonabsorbing media we obtain

$$
n(\xi) S_{0}(\xi)=\text { const }
$$

This allows us to simplify equation (5). In view of (8) we can go to the normalized Stokes parameters $P_{i}=S_{i} / S_{0}$ :

$$
\frac{d}{d \xi} \vec{P}=[\vec{P} * \vec{\omega}],
$$

where $\vec{P}=\left\{P_{1}, P_{2}, P_{3}\right\}$ is the normalized Stokes vector (pseudospin) and $\vec{\omega}=\left\{\omega_{1}, \omega_{2}, \omega_{3}\right\}$ - vector characterizing the local properties of the media. As we can see, (9) is analogous to the Bloch equations and describes the evolution of the light polarization state in an inhomogeneous anisotropic media with inhomogeneous gyrotropy. It is formally identical to the dynamics of spin in an external magnetic field [16] (or optical resonance in atoms [6]). Thus, the system of equations (4) and (9) allows us to fully analyze the spatial dynamics of the polarization state of light in media.

\section{ANALYSIS OF THE BEHAVIOR OF THE PSEUDOSPIN VECTOR.}

For further analysis we concretize permeability tensor of the media. Represent it as

$$
\varepsilon_{i j}=\left(\begin{array}{cc}
\varepsilon_{0}+\Delta \varepsilon(\xi) / 2 & i g(\xi) \\
-i g(\xi) & \varepsilon_{0}-\Delta \varepsilon(\xi) / 2
\end{array}\right) .
$$

Here part $\Delta \varepsilon(\xi)$ is induced by external influences inhomogeneous anisotropy (we consider homogeneous and isotropic media in nonperturbed state). Accordingly, $g(\xi)$ is induced inhomogeneous gyrotropy. For an arbitrary functional dependence of $\Delta \varepsilon(\xi)$ and $g(\xi)$ the system of equations (4) and (9) have not analytic solution. To identify the spatial dynamics of the polarization state of light one carry out the analytical solution when the spatial dependence of the induced inhomogeneity is the same as for $\Delta \varepsilon(\xi)$ and $g(\xi)$. Let

$$
\Delta \varepsilon(\xi)=\alpha_{0} f(\xi) ; g(\xi)=g_{0} f(\xi)
$$

\subsection{Coupled-wave method.}

From system (4) one can easily obtain

$$
\left\{\begin{array}{l}
\frac{d J_{x}}{d \xi}=i \frac{\alpha_{0} f(\xi)}{2 n_{0}} J_{x}-\frac{g_{0} f(\xi)}{2 n_{0}} J_{y} \\
\frac{d J_{y}}{d \xi}=\frac{g_{0} f(\xi)}{2 n_{0}} J_{x}-i \frac{\alpha_{0} f(\xi)}{2 n_{0}} J_{y}
\end{array}\right.
$$


By the substitution of $\tau=\left(2 n_{0}\right)^{-1} \int f(\xi) d \xi$ the system (11) is reduced to the equations with constant coefficients

$$
\left\{\begin{array}{l}
\frac{d J_{x}}{d \tau}(\tau)=i \alpha_{0} J_{x}(\tau)-g_{0} J_{y}(\tau) \\
\frac{d J_{y}}{d \tau}(\tau)=g_{0} J_{x}(\tau)-i \alpha_{0} J_{y}(\tau)
\end{array} .\right.
$$

The exclusion of $J_{y}(\tau)$ makes this system of equations of oscillator type $d^{2} J_{x}(\tau) / d \tau^{2}+\omega_{0}^{2} J_{x}(\tau)=0$. Here $\omega_{0}^{2}=\alpha_{0}{ }^{2}+$ $g_{0}^{2}$. The solution of this equation is well known

$$
\begin{gathered}
J_{x}(\xi)=A \cos \left\{\frac{\omega_{0}}{2 n_{0}} \int_{0}^{\xi} f(\xi) d \xi\right\}+B \sin \left\{\frac{\omega_{0}}{2 n_{0}} \int_{0}^{\xi} f(\xi) d \xi\right\}, \\
J_{y}(\xi)=\left(i \frac{\alpha_{0} A}{g_{0}}-\frac{\omega_{0} B}{g_{0}}\right) \cos \left\{\frac{\omega_{0}}{2 n_{0}} \int_{0}^{\xi} f(\xi) d \xi\right\}+\left(i \frac{\alpha_{0} A}{g_{0}}+\frac{\omega_{0} B}{g_{0}}\right) \sin \left\{\frac{\omega_{0}}{2 n_{0}} \int_{0}^{\xi} f(\xi) d \xi\right\} .
\end{gathered}
$$

The unknown constants are determined from boundary conditions. In the case of linear polarized input wave $J_{x}(0)=1$, $J_{y}(0)=0$ and respectively $=1, B=i \alpha_{0} / \omega_{0}$. In the case of circular polarization of input wave $-J_{x}(0)=1 / \sqrt{2}$, $J_{y}(0)=i / \sqrt{2}$ and $\mathrm{A}=1 / \sqrt{2}, B=i\left(\alpha_{0}-g_{0}\right) / \sqrt{2} \omega_{0}$. Thus, the expressions (13) completely solve our problem and characterize the spatial dynamics of the polarization state of light in a media with induced inhomogeneous anisotropy and gyrotropy. Fig.1 graphically shows evolution of $\left|E_{y}(\xi)\right|^{2}$ for constant $\alpha_{0}$ and three different values of the induced gyrotropy, and Fig. $2-\left|E_{y}(\xi)\right|^{2}$ dependence from $\xi$ for constant $g_{0}$, and for three different values of the induced anisotropy.

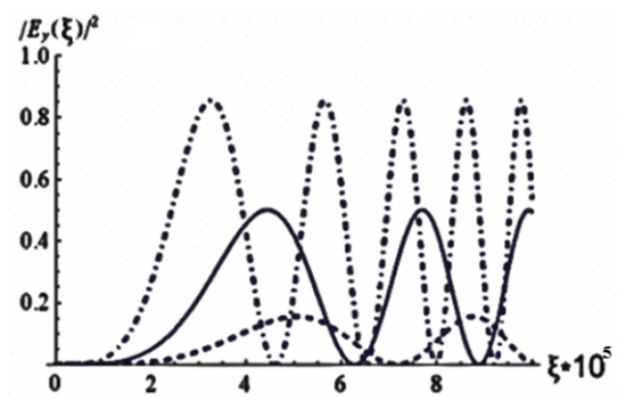

Fig.1. The results of numerical simulations of $\left|E_{y}(\xi)\right|^{2}$. The parameter values are $\epsilon_{0}=2.415 ; \alpha_{0}=3.5 * 10^{-11}$. The dashed curve is $-g_{0}=1.5 * 10^{-11}$, the solid curve is $g_{0}=3.5 * 10^{-11}$, the dotteddashed curve is $-g_{0}=$ $8.5 * 10^{-11}$.

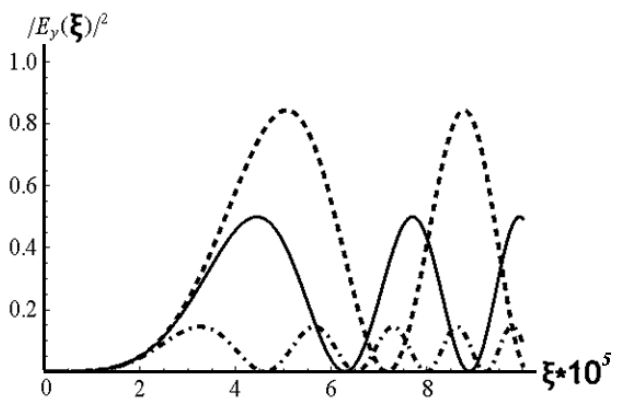

Fig.2. The results of numerical simulations of $\left|E_{y}(\xi)\right|^{2}$. The parameter values are $\epsilon_{0}=2.415 ; g_{0}=3.5 *$ $10^{-11}$. The dashed curve is $-\alpha_{0}=1.5 * 10^{-11}$, the solid curve is- $\alpha_{0}=3.5 * 10^{-11}$, the dotted dashed curve is $\alpha_{0}=8.5 * 10^{-11}$. 
Comparison of these figures shows that, depending on which parameter is varied, the character of the changes varies considerably. Namely, both in the first (Fig.1) and in the second (Fig.2) cases, the frequency of spatial oscillations increases, however the spatial behavior of the amplitude differs. For a fixed $\alpha_{0}$ oscillation amplitude increases with $g_{0}$ (Fig.1). For the second case (Fig.2), for fixed $g_{0}$ the oscillation amplitude decreases with increase of $\alpha_{0}$. It is not difficult to plot the amplitude spatial dependence for the case when we have circularly polarized wave at the entry of media. It is presented in Fig.3. "Resonance" is observed in the amplitude dependence. It has maximum when the "velocity" of changes of anisotropy and gyrotropy are the same. It is noteworthy that the numerical integration of (2) fully confirms the findings.

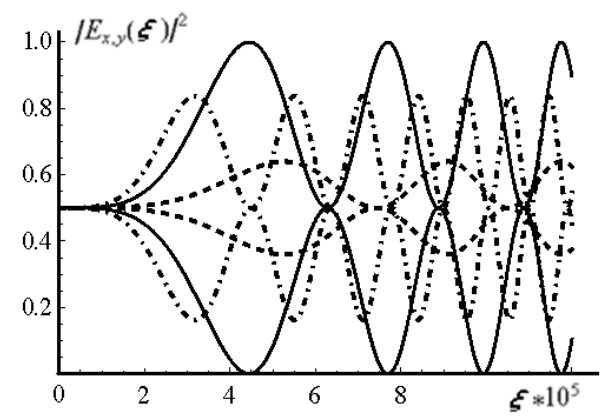

Fig.3. The results of numerical simulations for $\left|E_{x, y}(\xi)\right|^{2}$ with a circularly polarized light. The upper graphs correspond to $\left|\boldsymbol{E}_{\boldsymbol{x}}(\xi)\right|^{2}$, the bottom to $\left|\boldsymbol{E}_{\boldsymbol{y}}(\xi)\right|^{2}$. The parameter values are $\epsilon_{0}=2.415 ; \alpha_{0}=3.5 * 10^{-11}$. The dashed curve is - $g_{0}=0.5 * 10^{-11}$, the solid curve is- $g_{0}=3.5 * 10^{-11}$, the dashed curve is - $g_{0}=9 * 10^{-11}$.

\subsection{Pseudospin method.}

Let us analyze the problem using the Bloch equations. From (9), using (6) and (10a) one have

$$
\left\{\begin{array}{c}
\frac{d}{d \tau} P_{1}(\tau)=-g_{1} P_{2}(\tau) \\
\frac{d}{d \tau} P_{2}(\tau)=g_{1} P_{1}(\tau)+\alpha_{1} P_{3}(\tau) \\
\frac{d}{d \tau} P_{3}(\tau)=-\alpha_{1} P_{2}(\tau)
\end{array}\right.
$$

where $\alpha_{0} / n_{0}{ }^{3}=\alpha_{1} ; g_{0} / n_{0}{ }^{3}=g_{1}$, a $\tau=\left(2 n_{0}\right)^{-1} \int f(\xi) d \xi$. By elementary transformations of the system it's again reduced to an equation of the oscillator type

$$
\frac{d^{2}}{d \tau^{2}} P_{2}(\tau)+\Omega_{0}^{2} P_{2}(\tau)=0
$$

where $\boldsymbol{\Omega}_{\mathbf{0}}{ }^{2}=\boldsymbol{\alpha}_{\mathbf{1}}{ }^{2}+\boldsymbol{g}_{\mathbf{1}}{ }^{2}$. The solution of this equation is well known. In the case of a circularly polarized input wave boundary conditions are as follows:

$$
P_{2}(\tau=0)=P_{1}(\tau=0)=0 ; P_{3}(\tau=0)=1
$$

The final solution is as follows:

$$
\begin{gathered}
P_{1}(\xi)=\frac{\alpha_{1} g_{1}}{\omega_{1}^{2}}\left\{\cos \left[\frac{\omega_{1}}{2 n_{0}} \int_{0}^{\xi} f(\xi) d \xi\right]-1\right\} \\
P_{2}(\xi)=\frac{\alpha_{1}}{\omega_{1}} \sin \left[\frac{\omega_{1}}{2 n_{0}} \int_{0}^{\xi} f(\xi) d \xi\right] \\
P_{3}(\xi)=\frac{\alpha_{1}^{2}}{\omega_{1}^{2}}\left\{\cos \left[\frac{\omega_{1}}{2 n_{0}} \int_{0}^{\xi} f(\xi) d \xi\right]+1\right\}
\end{gathered}
$$


It is easy to verify that $P_{1}{ }^{2}+P_{2}{ }^{2}+{P_{3}}^{2}=1$. As an example, Fig.4 shows the behavior of the pseudospin vector on the Poincare sphere in three cases. When the media hold only induced inhomogeneous gyrotropy and wave at the entry is linearly polarized, the pseudospin vector circles in the equatorial plane. In the case when there is only induced inhomogeneous anisotropy, the rotation of the pseudospin vector in the meridional plane is observed. Both in the first, and in the second case because of inhomogeneity presence spatial frequency of rotation increases [14]. In the case of the simultaneous presence of inhomogeneous anisotropy and gyrotropy pseudospin vector circles on the cone surface.

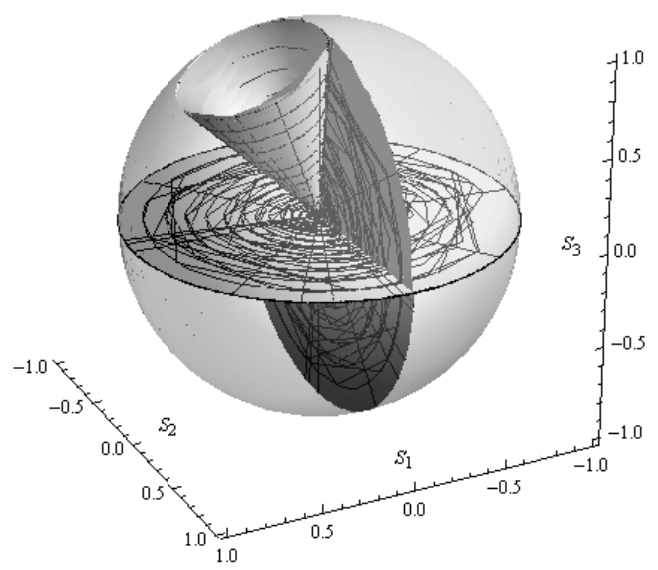

Fig.4. The behavior of the pseudospin vector on the Poincare sphere. Values parameters are as follows:

$$
\epsilon_{0}=2.415 ; \alpha_{0}=3.5 * 10^{-11} ; g_{0}=3.5 * 10^{-11} \text {. }
$$

For comparison it is possible to consider also behavior of a pseudospin vector in case of light polarization adiabatic transformation (Fig.5). As it is well known (see [13]), a move of pole of Poincare sphere (the input wave is circular polarized) pseudospin vector to the equator, leads to the transformation of polarization state to the linear polarized wave.

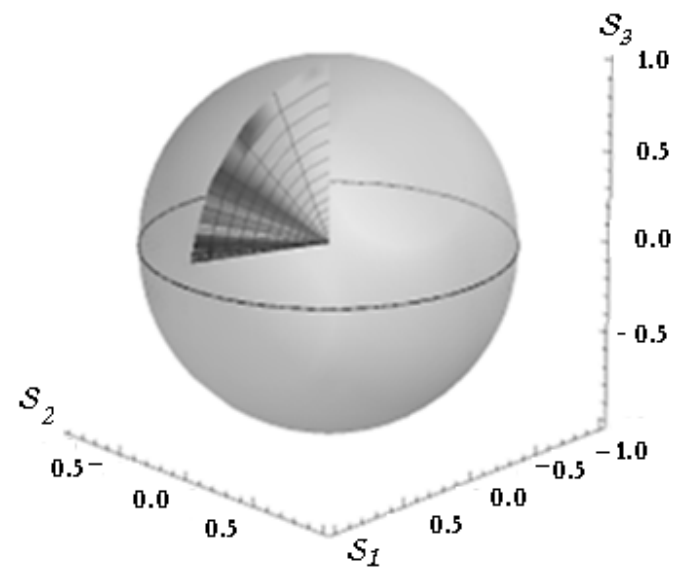

Fig.5. The behavior of the pseudospin vector on the Poincare sphere in the case of achromatic adiabatic transformation. Parameter values are

$$
\epsilon_{0}=2.415 ; \alpha_{0}=3.5 * 10^{-11} ; g_{0}=10^{-4} \text { : }
$$




\section{CONCLUSION}

In this work the theoretical analysis of the spatial dynamics of the polarization state of light propagating in weakly inhomogeneous anisotropic media with inhomogeneous girotropy is performed. Investigation is carried out by the method of coupled wave, as well as by the pseudospin method. The results of calculations show that the light polarization state during propagation in such media undergoes oscillations and its amplitude is maximum, when the speed of changes of the dielectric permittivity and gyrotropy is the same.

\section{REFERENCES}

[1]. Tuchin V.V., Wang L. V., Zimnyakov D. A., [Optical polarization in biomedical applications], (Springer), Berlin Heidelberg,(2006).

[2]. Bliokh K. Yu., Frolow D. Kravtsov Yu.,"Non-abelian evolution of electromagnetic waves in weakly anisotropic inhomogemeous medium", Phys. Rev.A, 75, 053821 (2007).

[3]. Simoni F., [Nonlinear optical properties of liquid crystals and polymer dispersed liquid crystals], World Scientific, Singapore, (1997).

[4]. Scharf T., [Polarized light in liquid crystals and polymers], A John Wiley and sons, N.Y.,(2006).

[5]. Fermi E., [Notes on quantum mechanics], The University of Chicago Press, (1965).

[6]. Allen L. and Eberly J., [Optical Resonance and Two-Level Atoms], A John Wiley and sons, N.Y.,(1975).

[7]. Kubo H., Nagata R. "Vector representation of polarized light in weakly inhomogeneous medium with briefringence and dichroism", JOSA, 73, 1719-1724, (1983).

[8]. Zapasskii V.S., Kozlov G.G., Polarized light in anisotropic medium and spin in a magnetic field, PhysicsUspekhi, 169, 909-915, (1999).

[9]. Rangelov A.A., Gaubats U., Vitanov N.V.,"Broadband conversation of light polarization”, Opt. Comm., 283 3891-3894, (2010).

[10]. Rangelov A A, "An achromatic polarization retarder realized slowly vaying linear and circular briefringence”.arXiv: 1105.0316v1, (2011);Rangelov A.A.,"A broadbend polarization retarde”,arXiv: 1104.4963v1, (2011).

[11]. Darsht M. J., Zeldovich B. Ya., Kundikova N. D., "Adiabatic following state of polarization of light for a smooth change in the medium", Optics and spectroscopy. 82, 660-662,(1997).

[12]. Aslanyan L. S., Grigoryan N. S., Nazaryan S. K. Circularly polarized light in weakly inhomogeneous anisotropic media with gyrotropy. Modern Problems in Optics and Photonics, Yerevan, Armenia, 27 August-2 September, 2009, Gitutyun Publ., p.109-113, (2009).

[13]. Aslanyan, L.S. Grigoryan N.S., Nazaryan S.K. Evolution of the polarization state of light in weakly inhomogeneous anisotropic media with gyrotropy. Proceedings of the conference "Lasers 2008". Ashtarak, Armenia, 1417 October, 2008, Gitutyun Publ.,p.58-61, (2009).

[14]. Vitanov N. V., Garraway B. M., "Landau-Zener model: Effects of finite coupling duration", Phys. Rev. A, 53, 4288-4304, (1996).

[15]. Genov G. T., Rangelov A. A., Vitanov N. V., "Propagation of light polarization in a birefringent medium: Exact analytic models",arXiv: 1102.1315v1, (2011).

[16]. Abragam A., [The principles of nuclear magnetism], Clarendon Press, Oxford, (1961). 